\title{
Generalized Gibbs ensemble and work statistics of a quenched Luttinger liquid
}

\author{
Balázs Dóra, ${ }^{1,2, *}$ Ádám Bácsi, ${ }^{2}$ and Gergely Zaránd ${ }^{1}$ \\ ${ }^{1}$ BME-MTA Exotic Quantum Phases Research Group, Budapest University of Technology and Economics, Budapest, Hungary \\ ${ }^{2}$ Department of Physics, Budapest University of Technology and Economics, Budapest, Hungary
}

(Received 14 March 2012; published 22 October 2012)

\begin{abstract}
We analyze the probability distribution function (PDF) of work done on a Luttinger liquid for an arbitrary finite duration interaction quench and show that it can be described in terms of a generalized Gibbs ensemble. We construct the corresponding density matrix with explicit intermode correlations, and determine the duration and interaction dependence of the probability of an adiabatic transition and the PDF of nonadiabatic processes. In the thermodynamic limit, the PDF of work exhibits a non-Gaussian maximum around the excess heat, carrying almost all the spectral weight. In contrast, in the small system limit most spectral weight is carried by a delta peak at the energy of the adiabatic process, and an oscillating PDF with dips at energies commensurate to the quench duration and with an exponential envelope develops. Relevance to cold atom experiments is also discussed.
\end{abstract}

DOI: 10.1103/PhysRevB.86.161109

PACS number(s): 05.30.Jp, 05.70.Ln, 67.85.-d, 71.10.Pm

Introduction. Nonequilibrium many-body dynamics constitutes a largely unexplored topic in comparison to its equilibrium counterpart. Its exploration has begun recently by a series of experiments on cold atomic gases ${ }^{1-4}$ and other systems,${ }^{5}$ triggering valuable theoretical works. ${ }^{6,7}$ A number of interesting issues has been analyzed, such as thermalization and equilibration and their relation to integrability, defect, and entropy production due to universal near adiabatic dynamics, quantum fluctuation relations, ${ }^{8}$ nonlinear response, etc.

Monitoring nonadiabatic dynamics provides a great deal of information about the universal features of the quantum system at hand. The scaling of expectation values or the first few moments of observables (e.g., the defect density) after a quench through a quantum critical point can be expressed in terms of the equilibrium critical exponents. ${ }^{6,7}$ However, the full characterization of a quantum state is only possible through its all higher moments, encoding unique information about nonlocal correlations of arbitrary order and entanglement. ${ }^{9}$ This is equivalent to determining the full distribution function of the quantity of interest. While its equilibrium evaluation is already rather involved, ${ }^{9}$ obtaining the full nonequilibrium distribution function of a physical observable has rarely been carried out. ${ }^{10}$

A fascinating exception is the statistics of work done during a quench, which has been studied in Refs. 11 and 12 for a sudden quench between gapped phases, separated by a quantum critical point (and gap closing). The probability distribution function (PDF) of work done, $P(W)$, involves all possible moments of energy, ${ }^{8}$ thus providing us with full characterization of the energy distribution.

While the transition between two gapped phases is of great interest, many interacting one-dimensional (1D) systems form gapless Luttinger liquid (LL) states. ${ }^{13}$ In particular, interacting cold atoms in a one-dimensional trap, e.g., often form such LLs, as also confirmed by experiments, ${ }^{2,3,14-16}$ but LL states appear in various spin models or interacting fermion systems. ${ }^{13}$ This state of matter is characterized by bosonic collective modes as elementary excitations, and by especially strong quantum fluctuations. How this system reacts to a time-dependent protocol, i.e., a quantum quench, is a highly nontrivial problem, though some of its properties have already been analyzed. ${ }^{17-22}$
Here we shall study the PDF of work on this prototypical example of a Luttinger liquid, determine $P(W)$ after an arbitrary quench protocol, and also construct explicitly the generalized Gibbs ensemble which reproduces all moments of $P(W)$. We remark that this is one of the rare occasions where the generalized Gibbs ensemble can be constructed analytically for an interacting model. The study of an arbitrary quench protocol is inspired by the observation that, in reality, quenches are neither completely adiabatic nor instantaneous and - as we demonstrate through the properties of $P(W)$ - the characteristic quench time is a crucial parameter of the quench itself. The work PDF is found to exhibit several universal forms (Gumbel or exponential distribution, for example) as controlled by the system size and interaction-dependent manybody orthogonality exponent $\alpha$ and the duration of the quench.

Hamiltonian. We consider an inherently gapless system of hard-core bosons (or an initially noninteracting Fermi gas) in one dimension, which is interaction quenched by a given protocol into a final LL liquid state. The corresponding LL Hamiltonian reads ${ }^{13,20}$

$$
H=\sum_{q \neq 0} \omega_{q}(t) b_{q}^{+} b_{q}+\frac{g_{q}(t)}{2}\left[b_{q} b_{-q}+b_{q}^{+} b_{-q}^{+}\right] .
$$

Here $\omega_{q}(t)=v(t)|q|$, and $v(t)=v+\delta v Q(t)$, with $v$ the bare "sound velocity," $\delta v$ its renormalization arising from interaction, and $b_{q}^{+}$the creation operator of a bosonic density wave. The interaction $g_{q}(t)=g_{2}(q)|q| Q(t)$ and the velocity are changed within a quench time $\tau$, with the quench protocol $Q(t)$ satisfying $Q(t<0)=0$ and $Q(\tau<t)=1$. For a linear quench, in particular, $Q(0<t<\tau)=t / \tau$. Equation (1) constitutes the effective model for bosons quenched away from the hard-core limit as well as for fermions quenched away from the noninteracting limit, or for an $X X Z$ spin chain, ${ }^{13,20}$ though our findings apply to interacting initial states as well. ${ }^{23}$

Since Eq. (1) is quadratic, the time evolution can be formally determined exactly. From the Heisenberg equation of motion, we obtain ${ }^{20}$

$$
b_{q}(t)=u_{q}(t) b_{q}(0)+v_{q}^{*}(t) b_{-q}^{+}(0),
$$


where the time dependence is carried by the time-dependent Bogoliubov coefficients $u_{q}(t)$ and $v_{q}(t)$, satisfying

$$
i \partial_{t}\left[\begin{array}{l}
u_{q}(t) \\
v_{q}(t)
\end{array}\right]=\left[\begin{array}{cc}
\omega_{q}(t) & g_{q}(t) \\
-g_{q}(t) & -\omega_{q}(t)
\end{array}\right]\left[\begin{array}{c}
u_{q}(t) \\
v_{q}(t)
\end{array}\right],
$$

with the initial condition $u_{q}(0)=1, v_{q}(0)=0$.

Generating function of work. Armed with the formal solution of the time-dependent Bogoliubov equations, Eq. (2), we analyze the statistics of work done. Albeit that the work done has been studied exhaustively in classical statistical mechanics, its quantum generalization has been carried out only recently, ${ }^{8}$ and its properties are known for very few systems. The quantum work cannot be represented by a single Hermitian operator ( $\Leftrightarrow$ not an observable), but rather its characterization requires two successive energy measurements, one before and one after the time-dependent protocol (thus work characterizes a process). The knowledge of all possible outcomes of such measurements yields the full probability distribution function (PDF) of work done on the system.

The characteristic function of work after the quench, $G(\lambda) \equiv \int d W e^{i W \lambda} P(W)$, can be expressed as ${ }^{8}$

$$
G(\lambda, \tau)=\left\langle\exp \left[i \lambda H_{H}(t>\tau)\right] \exp \left[-i \lambda H_{H}(0)\right]\right\rangle,
$$

where $H_{H}(t)$ is the Hamilton in the Heisenberg picture, and the expectation value is taken with the initial thermal state. For a sudden quench (SQ), $\tau=0$, and $G(\lambda, \tau)$ coincides with the Loschmidt echo. ${ }^{11}$ The expectation value, Eq. (4), is independent of $t$ for $t>\tau$, but depends on the quench protocol and its duration $\tau$. $H_{H}(t)$ is obtained by expressing the time-dependent boson operators in Eq. (1) using Eq. (2). Equation (4) can then be evaluated at $T=0$ upon realizing that the operators $K_{0}(q)=\left(b_{q}^{+} b_{q}+b_{-q} b_{-q}^{+}\right) / 2, K_{+}(q)=b_{q}^{+} b_{-q}^{+}$, $K_{-}(q)=b_{q} b_{-q}$ are the generators of a $\mathrm{SU}(1,1)$ Lie algebra, satisfying $\left[K_{+}(q), K_{-}(q)\right]=-2 K_{0}(q),\left[K_{0}(q), K_{ \pm}(q)\right]=$ $\pm K_{ \pm}(q)$, and the operators for distinct $q$ 's commute with each other. Using identities familiar from the theory of squeezing operators and the Baker-Campbell-Hausdorff relation, ${ }^{24}$ we get $^{23}$

$$
\ln G(\lambda, \tau)=i \lambda E_{\mathrm{ad}}-\sum_{q>0} \ln \left[1+n_{q}\left(1-e^{2 i \Omega_{q} \lambda}\right)\right],
$$

with $E_{\text {ad }}=E_{f}-E_{i}$ the difference between the adiabatic ground state energies in the final and initial state, $n_{q}=$ $\left\{\omega_{q}(t)-\Omega_{q}+2 \operatorname{Im}\left[v_{q}^{*}(t) \partial_{t} v_{q}(t)\right]\right\} / 2 \Omega_{q}$ the time-independent occupation number of mode $q$ in the final LL state, and $\Omega_{q}=\sqrt{\omega_{q}^{2}(t>\tau)-g_{q}^{2}(t>\tau)}$ the corresponding excitation energy. ${ }^{13}$

Generalized Gibbs ensemble. The fact that Eq. (5) depends only on the occupation numbers of the steady state indicates that a generalized Gibbs ensemble (GGE) may describe the final state. ${ }^{7}$ The analytic construction of the final density matrix is usually an inadmissible task. Therefore, one typically focuses only on few-body observables, and tries to build an approximate density matrix describing these. Such an approach is, however, unable to account for the complete PDF of work, which depends on all possible moments of energy.

In our case, the final Hamiltonian can be diagonalized by a Bogoliubov transformation giving $H_{f}=\sum_{q \neq 0} \Omega_{q} \hat{n}_{q}+E_{f}$. In the steady state $(t \gg \tau)$, the $\hat{n}_{q}$ 's and their arbitrary products are constants of motion, and therefore the density matrix of the GGE should be built up, in principle, from all of these operators. ${ }^{18,25}$ We find by intuition, however, that for a $T=0$ temperature quench the density operator

$$
\hat{\rho}_{G}=\frac{1}{Z_{G}} \prod_{q>0} \exp \left[-\beta_{q} \Omega_{q} \hat{n}_{q}\right] \delta_{\hat{n}_{q}, \hat{n}_{-q}}
$$

accounts for all intermode correlations in the final state. ${ }^{26}$ Here the mode-dependent inverse temperatures $\beta_{q}$ are defined through $n_{q} \equiv\left\langle\hat{n}_{q}\right\rangle \equiv 1 /\left[\exp \left(\beta_{q} \Omega_{q}\right)-1\right]$, and $Z_{G}=$ $\operatorname{Tr}\left\{\exp \left[-\sum_{q>0} \beta_{q} \Omega_{q} \hat{n}_{q}\right]\right\}$. Indeed, it is easy to show that $\operatorname{Tr}\left\{\hat{\rho}_{G} e^{i \lambda\left(H_{f}-E_{i}\right)}\right\}$ reproduces $G(\lambda, \tau)$ and thus the complete work distribution. ${ }^{23}$ Moreover, it gives back the expectation value of any operators in the steady state. Notice that the delta functions in $\hat{\rho}_{G}$ imply perfect correlations between the mode pairs $\pm q$, and the density matrices of Refs. $17,18,27$, and 28 are unable to reproduce the work statistics since they ignore these correlations.

The structure of Eq. (6) follows from the observation that, while time evolution does not conserve the number of bosons in a given pair of modes $\pm q$, it preserves $\hat{n}_{q}(t)-\hat{n}_{-q}(t)$. Since the only nonzero element of the initial density matrix corresponds to $\hat{n}_{q}=\hat{n}_{-q}=0$ at zero temperature, this can only evolve along the diagonal "direction," $\hat{n}_{q}(t)-\hat{n}_{-q}(t)=0$. The assumption that evolution during the quantum quench thermalizes the energy distribution of a given momentum pair with this constraint then amounts to the density matrix, Eq. (6). A given pair of modes thus thermalizes only along the diagonal of the density matrix, $\hat{n}_{q}=\hat{n}_{-q}$, characterized by an effective inverse temperature $\beta_{q}$, while the weight of the nondiagonal states $\hat{n}_{q} \neq \hat{n}_{-q}$ remains zero, as in the initial state. Though umklapp processes may lead to further thermalization at larger time scales, this structure is expected to be stable within experimental time scales. ${ }^{23}$

Perturbative generating function. Though an exact solution is formally also possible, the general properties of the final work PDF are already captured by a more transparent perturbative solution of Eq. (3). ${ }^{20}$ We thus expand Eq. (5) for small $g_{2}(q)$ and $\delta v$, and get for large system sizes $L$

$$
\begin{aligned}
\ln G(\lambda, \tau)= & i E_{\text {ad }}\left(\lambda-\int_{0}^{\tau} \int_{0}^{\tau} d t_{1} d t_{2} Q^{\prime}\left(t_{1}\right) Q^{\prime}\left(t_{2}\right) \tau_{0}\right. \\
& \left.\times\left[f\left(t_{1}-t_{2}+\lambda\right)-f\left(t_{1}-t_{2}\right)\right]\right) .
\end{aligned}
$$

Here $E_{\text {ad }}=-(L / v)\left(g_{2} / v \tau_{0}\right)^{2} / 16 \pi+\cdots<0$ and $f(t)=$ $\tau_{0} /\left(t+i \tau_{0}\right)$, with $\tau_{0}$ a short time cutoff associated with the finite range of interaction, $g_{2}(q)=g_{2} \exp \left(-\tau_{0} v|q|\right)$. Interestingly, the velocity renormalization $\delta v$ does not enter to lowest order. The cumulants $C_{n}$ of the work done can be derived by expanding Eq. (7) in $\lambda$ (see Ref. 23).

Work PDF: Generic properties. To analyze the PDF of work it is worth introducing the dimensionless work, measured with respect to the adiabatic ground state energy shift,

$$
w \equiv\left(W-E_{\mathrm{ad}}\right) /\left|E_{\mathrm{ad}}\right| .
$$

The distribution of $w$ is then obtained by Fourier transforming $G(\lambda, \tau)$ as

$$
p(w)=\mathcal{P}_{\text {ad }} \delta(w)+\rho(w) .
$$


The Dirac-delta peak corresponds to the probability of staying in the adiabatic ground state, while the broad structure $\rho(w)$ is associated with transitions to excited states with $w>0$. The weight $\mathcal{P}_{\text {ad }}$ can be expressed as

$$
\ln \left(\mathcal{P}_{\mathrm{ad}}\right)=-i \alpha \int_{0}^{\tau} \int_{0}^{\tau} d t_{1} d t_{2} Q^{\prime}\left(t_{1}\right) Q^{\prime}\left(t_{2}\right) f\left(t_{1}-t_{2}\right) .
$$

The prefactor $\alpha=\left|E_{\mathrm{ad}} \tau_{0}\right| \sim N\left(g_{2} / v\right)^{2}$ denotes the total angle of Bogoliubov rotations ( $N \sim L / v \tau_{0}$ is the number of particles), and can be viewed as the many-body orthogonality exponent. It is also closely related to the fidelity susceptibility. ${ }^{29}$ Alternatively, we can rewrite it as $\alpha \sim L / l$, with $l$ the mean free path. Thus $\alpha \gtrless 1$ describes, using fidelity nomenclature, the thermodynamic or small system limits ${ }^{29}$ or, alternatively, corresponds to the diffusive or ballistic limits, respectively, depending on the picture used.

In the adiabatic limit $\left[\tau \rightarrow \infty\right.$ with $Q^{\prime}(t) \rightarrow 0$ for all $\left.t\right]$, a finite system always stays in its ground state, and the time evolved wave function coincides with the lowest energy eigenfunction of the instantaneous Schrödinger equation. ${ }^{30}$ Consequently, only the first term remains in Eq. (9) with $\mathcal{P}_{\text {ad }}=1$. For $\tau \ll \tau_{0}$, on the other hand, $\mathcal{P}_{\text {ad }}$ scales as $\sim \exp (-\alpha) \sim$ $\exp (-\operatorname{cst} L)$ (see Fig. 1), and in the limit $L \rightarrow \infty$-but fixed interaction $-\mathcal{P}_{\text {ad }}$ vanishes due to the orthogonality catastrophe.

Sudden quench $(S Q)$ limit. In the extreme limit of a SQ, $\tau \ll$ $\tau_{0},{ }^{31} G(\lambda, \tau)$ simplifies to $G(\lambda)=\exp \left[i E_{\mathrm{ad}} \lambda^{2} /\left(\lambda+i \tau_{0}\right)\right]$, and the continuum part of the PDF of work is evaluated exactly as

$$
\rho_{\mathrm{SQ}}(w)=\mathcal{P}_{\mathrm{ad}} \exp (-\alpha w) \alpha w^{-1 / 2} I_{1}(2 \alpha \sqrt{w}),
$$

with $\mathcal{P}_{\text {ad }}=\exp (-\alpha)$ and $I_{1}(x)$ the modified Bessel function of the first kind. This is the noncentral $\chi^{2}$ distribution with noncentrality parameter $4 \alpha$ in the limit of zero degrees of freedom. ${ }^{32}$ The average work is zero, ${ }^{20}$ since for a SQ the system remains in its initial state and-on average - there is no back reaction. Entropy is, however, generated by populating high and low energy configurations.
The shape of $\rho(w)$ depends crucially on the orthogonality parameter $\alpha$. The thermodynamic limit $\alpha \gg 1$ reveals universal behavior: Almost all probability weight is carried by a peak centered at around $W=0(w=1)$ and of width $\Delta W \sim\left|E_{\mathrm{ad}}\right| / \sqrt{\alpha}$,

$$
\rho_{\mathrm{SQ}}^{\alpha \gg 1}\left(w \gg \alpha^{-2}\right) \approx \frac{\exp \left(-\alpha[1-\sqrt{w}]^{2}\right)}{w^{3 / 4} \sqrt{4 \pi \alpha^{-1}}},
$$

whose high energy tail decays according to the gamma distribution, $\sim \exp (-\alpha w) / w^{3 / 4}$. In the small system regime $\alpha \ll 1$, on the other hand, the delta function retains almost all weight, and transfers only a fraction $\sim \alpha$ to an exponential distribution of width $\Delta W \sim\left|E_{\text {ad }}\right| / \alpha$ and threshold at $E_{\text {ad }}$ for $w \ll \alpha^{-2}$. In the crossover regime, $\alpha \sim 1$, the maximum shifts to lower energies and the PDF of work develops a sizable value right above the threshold at $E_{\text {ad }}$ (see Fig. 1). The maximum of $P(W)$ occurs at $W>E_{\text {ad }}$ for $\alpha>2$, while the PDF becomes monotonically decreasing for $\alpha<2$.

Finite quench times. For finite duration quenches, in addition to the orthogonality parameter $\alpha$, the work statistics also depends on $\tau$ and the protocol $Q(t)$ itself. For definiteness, we focus here on a linear quench, ${ }^{23}$ and measure the degree of adiabaticity by $\tilde{\tau}=\tau / \tau_{0}$.

For a finite duration quench, $\tilde{\tau}>1$, only a fraction $1 / \tilde{\tau}$ of the excitations experiences the quench as sudden. Consequently, in the expression of $\mathcal{P}_{\text {ad }}$, the orthogonality exponent $\alpha$ is replaced by $\alpha_{\tau} \sim \alpha / \tilde{\tau}$, and $\mathcal{P}_{\text {ad }}$ becomes a monotonously increasing function of $\tilde{\tau}$ (see Fig. 1). The crossover with increasing $\alpha_{\tau}$ from $\mathcal{P}_{\text {ad }} \lesssim 1$ to vanishingly small spectral weight, $\mathcal{P}_{\text {ad }}$, occurs at $\alpha \sim \tilde{\tau}$.

Close to the threshold, $W-E_{\text {ad }} \ll 1 / \tau$, only states with energy smaller than $1 / \tau$ and thus feeling a SQ contribute to work. Therefore, apart from a normalization factor, the PDF of work agrees with the SQ result

$$
\rho\left(w \ll \alpha^{-1} \tilde{\tau}^{-1}\right) \approx \mathcal{P}_{\mathrm{ad}} \exp (\alpha) \rho_{\mathrm{SQ}}(w),
$$

and depends on $\tau$ only through $\mathcal{P}_{\text {ad }}$.
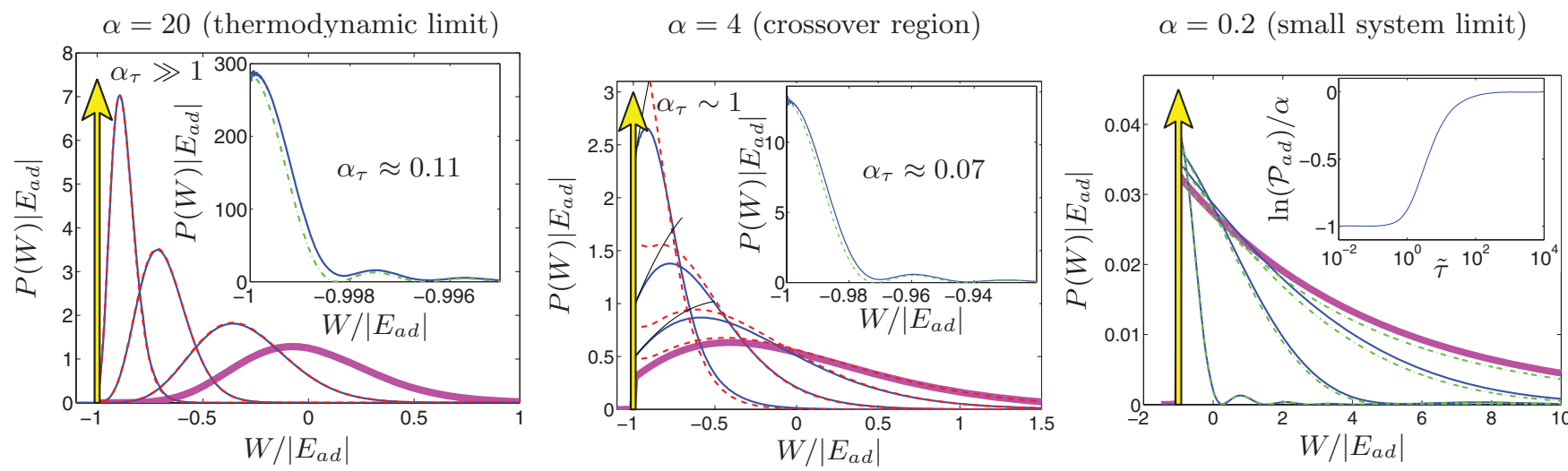

FIG. 1. (Color online) The PDF of work done on a LL is plotted after a linear quench from the numerical evaluation of Eq. (7) (blue solid line). Left: $\alpha=20$ with $\tilde{\tau}=0,1,2.5$, and 5 from right to left and 180 [inset, $P\left(W>E_{\text {ad }}\right)$ only]; middle: $\alpha=4$ with $\tilde{\tau}=0,1,2$, and 4 with increasing peak height and 55 (inset); right: $\alpha=0.2$ with $\tilde{\tau}=0,2,5$, and 25 from right to left. The thick magenta line denotes the exact SQ expression [Eq. (11)], the red dashed line represent Eq. (15), the thin black line in the middle panel visualizes Eq. (13), while the green dashed-dotted line shows Eq. (14). The vertical arrow at $W=E_{\text {ad }}$ denotes the Dirac-delta peak, whose spectral weight $\mathcal{P}_{\text {ad }}$ is shown in the inset of the right panel on semilog scale as a function of the ramp time $\tau$. 
For $\tilde{\tau} \gg 1$, however, Eq. (13) describes only a small region close to $E_{\text {ad }}$ (see thin black lines in Fig. 1), and the overall shape depends both on $\alpha$ and $\tilde{\tau}$. For $4 \alpha \gg \tilde{\tau}$, almost all spectral weight is carried by the nonadiabatic processes $[\rho(w)]$ around the typical value $W_{\text {typ }}-E_{\text {ad }} \sim 2\left|E_{\text {ad }}\right| \ln (\tilde{\tau}) / \tilde{\tau}^{2}$, clearly separated from the adiabatic process. For $\tilde{\tau} \gg 4 \alpha$, the adiabatic process gains spectral weight, $\mathcal{P}_{\text {ad }} \approx 1$, but a maximum for $W>E_{\text {ad }}$ remains present, though it gradually merges with the adiabatic processes.

In particular, in the small system limit $\alpha_{\tau} \ll 1$, e.g., we can expand Eq. (7) to get

$$
\rho(w) \approx \mathcal{P}_{\mathrm{ad}} \alpha^{2}\left(\frac{\sin [w \alpha \tilde{\tau} / 2]}{w \alpha \tilde{\tau} / 2}\right)^{2} \exp (-\alpha w) .
$$

Thus, for a linear quench, for energies commensurate with the quench time the PDF is zero (see Fig. 1). This is related to the steady state behavior of the occupation numbers, $n_{q} \approx\left[g_{2}(q) \sin (v|q| \tau) / 2 v^{2}|q| \tau\right]^{2}$ for $g_{2} \ll v,{ }^{23}$ reflecting that modes with energy commensurate to the quench time stay almost unoccupied at $T=0$. In this limit $(\alpha \ll \tilde{\tau}$ and $\tilde{\tau} \gg 1$ ), the system evolves almost adiabatically, nonadiabatic processes have only a small probability $\sim \alpha / \tilde{\tau}$, and the typical work done in the case of a rare nonadiabatic process is $W_{\text {typ }} \approx-\alpha^{2} \pi / \tau$.

Increasing $\alpha$, the zeros of the PDF turn gradually into dips, and the PDF develops a more universal form. In the thermodynamic limit $\alpha_{\tau} \gg 1$, using the method of steepest descent we obtain

$$
\rho(w) \approx \frac{\mathcal{P}_{\mathrm{ad}} \tilde{\tau}^{3 / 2} \sqrt{\alpha}}{2 \sqrt{\tan ^{3}(s) \pi}} \exp \left[w\left(\frac{\tilde{\tau}^{2}}{2}-\alpha\right)+2 \frac{\alpha s}{\tilde{\tau}}\right]
$$

for $\alpha s \gg \tilde{\tau}$, with $s \equiv \arctan \left[\sqrt{\exp \left(w \tilde{\tau}^{2}\right)-1}\right]$. For $w \gg$ $1 / \tilde{\tau}^{2} \gg 1 / \alpha^{2}, \rho(w)$ in Eq. (15) behaves as a generalized Gumbel distribution of index $a=\frac{1}{2}+\frac{2 \alpha}{\tilde{\tau}^{2}} \cdot 33$

This latter emerges in the context of global fluctuations, describing the limit distribution of the $a$ th maximum of a sequence of independent and identically distributed random variables. ${ }^{9}$ The distribution in the $1 / \tilde{\tau}^{2} \gg w \gg 1 / \alpha^{2}$ region resembles closely to Eq. (12) apart from its normalization.

Experimental relevance. Our results can be tested on onedimensional hard-core bosons ${ }^{34}$ or noninteracting fermions as initial states. The detection of the PDF of work requires two energy measurements, one before and one after the time-dependent protocol. The first energy measurement can be omitted if we prepare the initial wave function in an energy eigenstate of $H(t=0)$. The resulting energy distribution can then be probed using time-of-flight experiments, ${ }^{4,7}$ similarly to Ref. 35. The crossover between the various regimes can be monitored by tuning $\tau / \tau_{0}$ and $\alpha \sim N\left(g_{2} / v\right)^{2}$, where $N$ is the number of particles in a $1 \mathrm{D}$ trap, typically with $N \sim 10^{2}-10^{3}$ atoms. ${ }^{2,3,16}$ By choosing $g_{2} / v \sim 1 / \sqrt{N}, \alpha$ becomes of order unity, facilitating the observation of crossover between the various regimes. For one-dimensional interacting bosons (i.e., Bose-Hubbard model), $v \sim J$ and $g_{2} \sim J^{2} / U$ for $U \gg J$ (close to the hard-core boson limit) with $U$ the on-site interaction $^{36}$ and $J$ the hopping amplitude. By quenching away from the initial $U \gg J \Leftrightarrow g_{2} \approx 0$ limit (e.g., by changing the lattice parameters or tuning the Feshbach resonance), a final interaction $U \sim J \sqrt{N}$ is reachable. For weakly interacting fermions, $v \sim J$ and $g_{2} \sim U$, therefore ramping from the weakly interacting case to $U \sim J / \sqrt{N}$ is desirable. Nonetheless, our results apply also to interacting initial states. ${ }^{23}$

Summary. We have studied the PDF of work done on a LL after an interaction quench, realizable in strongly interacting Bose systems. We have constructed the density matrix of the generalized Gibbs ensemble with intermode correlations, describing arbitrary correlations of the steady state, thus the PDF of work. The PDF exhibits markedly different characteristics depending on the system size, quench duration, and interaction strength. Our method is applicable to the full PDF of other observables as well, e.g., density fluctuations. ${ }^{37}$ We also emphasize that our results in Eqs. (5) and (6) apply also to a variety of other systems with effective bosonic Hamiltonians as in Eq. (1), including interacting higher dimensional bosons or spin systems within a spin-wave theory.

Acknowledgment. This research has been supported by the Hungarian Scientific Research Funds No. K72613, No. K73361, No. K101244, No. CNK80991, and No. TÁMOP4.2.1/B-09/1/KMR-2010-0002, and by the Bolyai program of the Hungarian Academy of Sciences and by the ERC Grant Nr. ERC-259374-Sylo. *dora@kapica.phy.bme.hu

${ }^{1}$ M. Greiner, O. Mandel, T. W. Hänsch, and I. Bloch, Nature (London) 419, 51 (2002).

${ }^{2}$ T. Kinoshita, T. Wenger, and D. S. Weiss, Nature (London) 440, 900 (2006).

${ }^{3}$ S. Hofferberth, I. Lesanovsky, B. Fischer, T. Schumm, and J. Schmiedmayer, Nature (London) 449, 324 (2007).

${ }^{4}$ I. Bloch, J. Dalibard, and W. Zwerger, Rev. Mod. Phys. 80, 885 (2008).

${ }^{5}$ Y. Utsumi, D. S. Golubev, M. Marthaler, K. Saito, T. Fujisawa, and G. Schön, Phys. Rev. B 81, 125331 (2010).

${ }^{6}$ J. Dziarmaga, Adv. Phys. 59, 1063 (2010).

${ }^{7}$ A. Polkovnikov, K. Sengupta, A. Silva, and M. Vengalattore, Rev. Mod. Phys. 83, 863 (2011).
${ }^{8}$ M. Campisi, P. Hänggi, and P. Talkner, Rev. Mod. Phys. 83, 771 (2011).

${ }^{9}$ V. Gritsev, E. Altman, E. Demler, and A. Polkovnikov, Nat. Phys. 2, 705 (2006).

${ }^{10}$ M. Gring, M. Kuhnert, T. Langen, T. Kitagawa, B. Rauer, M. Schreitl, I. Mazets, D. A. Smith, E. Demler, and J. Schmiedmayer, Science 337, 1318 (2012).

${ }^{11}$ A. Silva, Phys. Rev. Lett. 101, 120603 (2008).

${ }^{12}$ L. Campos Venuti and P. Zanardi, Phys. Rev. A 81, 022113 (2010).

${ }^{13}$ T. Giamarchi, Quantum Physics in One Dimension (Oxford University Press, Oxford, UK, 2004).

${ }^{14}$ E. Haller, R. Hart, M. J. Mark, J. G. Danzl, L. Reichsollner, M. Gustavsson, M. Dalmonte, G. Pupillo, and H.-C. Nagerl, Nature (London) 466, 597 (2010). 
${ }^{15}$ I. L. S. Hofferberth, T. Schumm, A. Imambekov, V. Gritsev, E. Demler, and J. Schmiedmayer, Nat. Phys. 4, 489 (2008).

${ }^{16}$ B. Paredes, A. Widera, V. Murg, O. Mandel, S. F. I. Cirac, G. V. Shlyapnikov, T. W. Hänsch, and I. Bloch, Nature (London) 429, 277 (2004).

${ }^{17}$ M. A. Cazalilla, Phys. Rev. Lett. 97, 156403 (2006).

${ }^{18}$ A. Iucci and M. A. Cazalilla, Phys. Rev. A 80, 063619 (2009).

${ }^{19}$ G. S. Uhrig, Phys. Rev. A 80, 061602(R) (2009).

${ }^{20}$ B. Dóra, M. Haque, and G. Zaránd, Phys. Rev. Lett. 106, 156406 (2011).

${ }^{21}$ E. Perfetto and G. Stefanucci, Europhys. Lett. 95, 10006 (2011).

${ }^{22}$ J. Rentrop, D. Schuricht, and V. Meden, New J. Phys. 14, 075001 (2012).

${ }^{23}$ See Supplemental Material at http://link.aps.org/supplemental/ 10.1103/PhysRevB.86.161109 for further technical details.

${ }^{24}$ D. R. Truax, Phys. Rev. D 31, 1988 (1985).

${ }^{25}$ D. M. Gangardt and M. Pustilnik, Phys. Rev. A 77, 041604 (2008).
${ }^{26}$ Strictly speaking, our GGE works for the diagonal elements (in $n_{q}$ 's) of the density matrix, and off-diagonal elements depend explicitly on time (Ref. 23), though their contribution dephases at $t \rightarrow \infty$.

${ }^{27}$ M. Rigol, V. Dunjko, V. Yurovsky, and M. Olshanii, Phys. Rev. Lett. 98, 050405 (2007).

${ }^{28}$ T. Barthel and U. Schollwöck, Phys. Rev. Lett. 100, 100601 (2008).

${ }^{29}$ M. M. Rams and B. Damski, Phys. Rev. Lett. 106, 055701 (2011).

${ }^{30}$ F. Pollmann, S. Mukerjee, A. G. Green, and J. E. Moore, Phys. Rev. E 81, 020101 (2010).

${ }^{31}$ In more realistic (e.g., multiband) models, other nonuniversal energy scales may play a role for a SQ.

${ }^{32}$ A. F. Siegel, Biometrika 66, 381 (1979).

${ }^{33}$ M. Clusel and E. Bertin, Int. J. Mod. Phys. B 22, 3311 (2008).

${ }^{34}$ T. Kinoshita, T. Wenger, and D. S. Weiss, Science 305, 1125 (2004).

${ }^{35}$ D. Chen, M. White, C. Borries, and B. DeMarco, Phys. Rev. Lett. 106, 235304 (2011).

${ }^{36}$ M. A. Cazalilla, R. Citro, T. Giamarchi, E. Orignac, and M. Rigol, Rev. Mod. Phys. 83, 1405 (2011).

${ }^{37}$ J. Armijo, T. Jacqmin, K. V. Kheruntsyan, and I. Bouchoule, Phys. Rev. Lett. 105, 230402 (2010). 